\title{
A novel technique for removing large gastric subepithelial tumors with ESD method in the subcardia region
}

\author{
BINGTUAN LIU ${ }^{1-3^{*}}$, HAN CHEN $^{1 *}$, WEIFENG ZHANG ${ }^{1,2^{*}}$ and GUOXIN ZHANG ${ }^{1}$ \\ ${ }^{1}$ Department of Gastroenterology, First Affiliated Hospital of Nanjing Medical University; \\ ${ }^{2}$ The First Clinical Medical College of Nanjing Medical University, Nanjing, Jiangsu 210029; \\ ${ }^{3}$ Department of Gastroenterology, Jiangsu Jiangyin People's Hospital, Wuxi, Jiangsu 214400, P.R. China
}

Received March 13, 2019; Accepted August 7, 2019

DOI: $10.3892 / 01.2019 .10894$

\begin{abstract}
Previously, patients with tumors larger than $4 \mathrm{~cm}$ in sub-cardia region usually received open gastrectomy. Due to its anatomic features, the cardia is often considered as a contraindicated area for endoscopic resection. Herein, we report a novel technique of endoscopic submucosal dissection (ESD) which facilitates the removal of gastric subepithelial tumors (SMTs) larger than $4 \mathrm{~cm}$ in the subcardia and fundus region. This is a retrospective case series of patients with SMTs larger than $4 \mathrm{~cm}$ in the subcardia and fundus regions who received the novel procedure of ESD between October 2015 and October 2016. The novel procedure of ESD involved a median linear incision of the mucosa being made in the central area of the tumor, followed by the submucosal dissection. The residual defect was finally closed using titanium endoclips. The endoscopical outcomes, histopathological findings as well as other complications were assessed. Eight patients fulfilled the entry criteria. The mean lesion size was $45.6 \pm 7.5 \mathrm{~mm}$ (range: $40.0-65.0 \mathrm{~mm}$ ), and the mean operating time was $83 \pm 13$ min (range: $60-100 \mathrm{~min}$ ). The en bloc resection rate was $100 \%$. Although perforations occurred in 5 out of 8 patients, they were successfully closed with endoclips. The median length of inpatient hospital stay was 6 days (range: 5-8 days). No patients needed further gastrectomy. The median follow-up was 36 months and none of the patients developed local recurrence or distant metastasis. The advanced procedure of ESD is feasible and safe for tumors more than $4 \mathrm{~cm}$ in the subcardia region. It could be applied as a novel technique for treating patient without surgical interventions.
\end{abstract}

Correspondence to: Dr Guoxin Zhang, Department of Gastroenterology, First Affiliated Hospital of Nanjing Medical University, 300 Guangzhou Road, Nanjing, Jiangsu 210029, P.R. China

E-mail: guoxinz@njmu.edu.cn; guoz57@126.com

${ }^{*}$ Contributed equally

Key words: gastric subepithelial tumors, subcardia region, endoscopic submucosal dissection

\section{Introduction}

Gastric subepithelial neoplasms usually present as protuberances covered by normal-appearing mucosa on endoscopy (1). These protuberances vary in different tumor types which include gastrointestinal stromal tumors (GISTs), lipomas, leiomyomas and liposarcomas (2). In western countries, subtotal gastrectomy or laparoscopic surgery is usually considered the mainstay treatment (3).

Recently, endoscopic submucosal dissection (ESD) has become a minimally invasive approach for gastric subepithelial lesions. The technique was first reported in 1988 for treating early gastric cancers in Japan (4), and now it has been widely used in Asian countries as an effective and relatively safe method for removing gastric tumors. Nonetheless, controversies still exist because this technique is also associated with high rates of resection failure, intraoperative perforation and excessive haemorrhage $(5,6)$, especially in cases of large tumors growing around the gastric cardia $(3,7)$. The gastric cardia is an anatomically narrow region in which the most proximal part of the stomach is attached to the esophagus (8). Due to its anatomic features, the cardia is often considered as a contraindicated area for endoscopic resections (9). In the routine ESD procedure, circumferential mucosal incision is initially applied, and lesion tissue will be subsequently retrieved $(9,10)$. If the tumor is large, it would be more technically difficult to finish endoscopic closure of the residual tissues, and operative time would be elongated accordingly.

Herein, we report an advanced ESD procedure which may facilitate the endoscopic resection techniques of removing large subepithelial tumors, especially for those in the gastric cardia region. We analyzed the short-term clinical outcomes to determine the feasibility of this approach.

\section{Patients and methods}

Study design and patients. The present study was performed according to the principles of the Declaration of Helsinki (1989). A prospective case series was conducted at the First Affiliated Hospital of Nanjing Medical University (Nanjing, China) from October 2015 to October 2016. Patients (3 males and 5 females) were enrolled to receive ESD using the advanced procedures if they met the following criteria: i) individuals aged 18-75 years, 
A

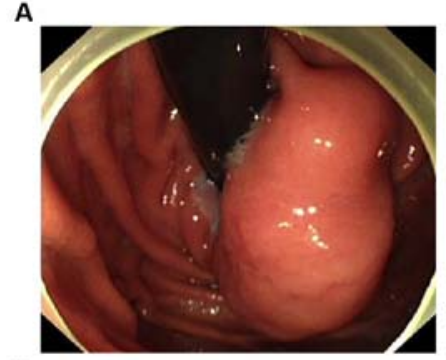

D
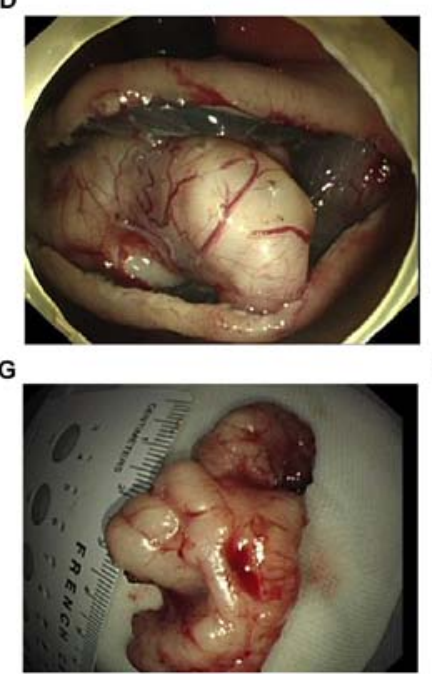

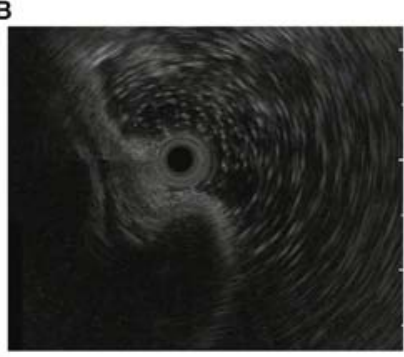

E
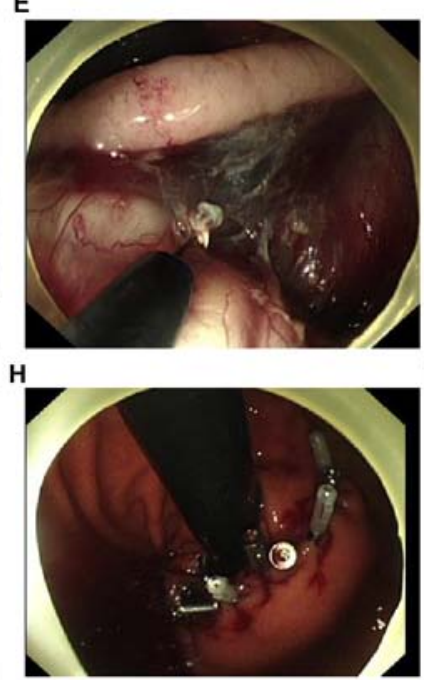

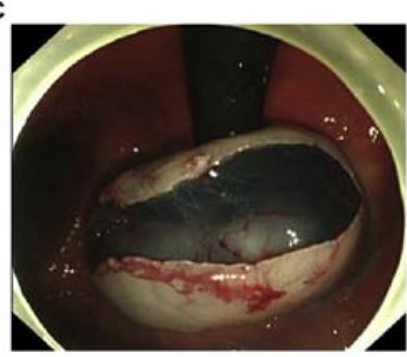

$\mathbf{F}$
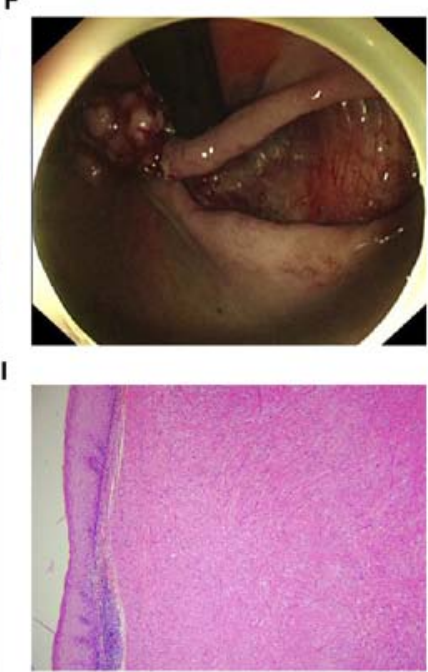

Figure 1. The advanced ESD procedure. A solution mixed with $0.9 \%$ saline, methylene blue and diluted epinephrine was injected into the submucosal layer of the tumor. A hook-knife was directly used to make a median linear incision of the mucosa in the position with the central, superficial mucosa area of the tumor. The incision was made to expose the body of the tumor in the center areas. Then the en bloc resection was carried out with the insulated-tip knife until the tumor was completely dissected. After the tumor was dissected, the residual defect was closed using titanium endoclips. (A) Endoscopic view of an SMT in the subcardia region of the stomach. (B) EUS images showing a narrow connection of tumor with the fourth hypoechoic EUS layer, indicating the origin from the superficial muscularis propria. (C) A median linear incision was made into the mucosa. (D) A submucosal dissection was made to expose the SMT. (E) Endoscopic dissection of the tumor. (F) Endoscopic view of residual defect after the body of the tumor dissection. (G) The residual defect was closed using endoclips. (H) View of tumor after dissection. (I) Histopathology of the tumor suggested leiomyomas. ESD, endoscopic submucosal dissection; SMT, subepithelial tumor; EUS, endoscopic ultrasonography.

ii) no contracted signs for endoscopical resection, iii) gastric subepithelial tumor invades the submucosal or the muscularis propria layer, iv) the diameter of tumor ranges from $4-8 \mathrm{~cm}$, and v) tumor located in the subcardia or fundus region and vi) no extra-gastrointestinal metastasis. Exclusion criteria were: i) patients with severe heart, liver and kidney dysfunction, ii) patients with abnormal coagulation function, iii) patients with tumor metastasis and iv) patients who refused endoscopic treatment. The diagnosis was established on the findings of gastroscopy and endoscopic ultrasonography (EUS). The maximum diameter of tumor and layer of tumor origin were carefully evaluated by two experienced GI endoscopists. Tumors were classified to have muscularis propria origin if they were connected with the underlying fourth layer in EUS (8). Patients underwent routine laboratory examinations in addition to electrocardiography and computed tomography (CT) scan before the ESD procedure. If the tumor was incompletely resected, patient would be referred to receive surgical interventions. The data of therapeutic outcomes of ESD such as the complete resection, bleeding, perforation and the operating time were analyzed.

The study was approved by the Ethics Committee of Nanjing Medical University. All enrolled patients signed an informed consent after receiving explanations of possible treatment options and potential complications regarding endoscopic treatments.

ESD procedures. All patients were in the left-lateral position and were under general anesthesia with an intravenous injection of remifentanil $(0.2 \mathrm{mg} / \mathrm{h})$ and propofol $(0.5 \mathrm{mg} / \mathrm{kg})$. ESD was performed by two experienced endoscopists. A singlechannel video endoscope was applied (Olympus GIT-H260) along with a transparent cap (Olympus D-201-12704) on the tip, providing a good view during the procedure. Fig. 1 summarizes the whole procedure of the method. The marking step was skipped in this procedure. To separate the mucosa and the tumor, a solution mixed with $0.9 \%$ saline, methylene blue and diluted epinephrine $(1: 10,000)$ was injected into the submucosal layer in position with the central, superficial mucosa area of the tumor. A hook-knife (model KD-620LR; Olympus) was directly used to make a median linear incision of the mucosa in the same position above. Next, the en bloc resection was carried out with the hook knife, or an insulatedtip knife (ITknife2 model KD-611L; Olympus) until the tumor was completely dissected: i) First, the submucosal tissues were dissected gradually from both sides of the incision; ii) next, a hook knife or an insulated-tip knife was used to carefully dissect the tumor at the bottom from its original layer. 
Muscular fibers or stalks were also resected to separate the tumor and the muscularis propria. If necessary, an additional submucosal injection was carried out repeatedly during the dissection procedures. Endoscopic full-thickness resection (EFR) was applied in cases where the tumor was attached tightly to the muscularis propria layer or the serosa (10): i) An active perforation was made around the tumor by the needle knife (KD-10Q-1, Olympus), ii) the full-thickness incision was applied by the insulated-tip (IT) knife (KD-610L, Olympus) and iii) if necessary, the forceps within the Dual-channel gastroscope were applied to prevent the tumor from dropping into the peritoneal cavity. After the tumor was dissected, the residual defect was closed using titanium endoclips (Sureclip, Micro-Tech). Hot hemostatic forceps (Olympus) were applied for hemostasis. When necessary, endoclips were used to stop bleeding. If a tension pneumoperitoneum developed due to the perforation, a 20-gauge needle was applied to make an immediate decompression of the peritoneal cavity. In general, patients were allowed liquids after $24 \mathrm{~h}$ of ESD. The daily use of phosgel and proton pump inhibitor were administered for two months after the procedure. The ESD related complications were recorded in all patients to evaluate the efficacy and safety of this advanced ESD method.

Histopathology. Resected samples were immersed in $10 \%$ formalin and then embedded in the paraffin with eosin and hematoxylin staining. Histopathological characteristics including the tumor size, resection margin status and invasion depth were carefully evaluated by two distinct pathologists. The complete resection was defined as resection margins free of any tumor tissues. Immunohistochemical staining was performed in all patients. GIST was diagnosed by the positive results of SMA, c-KIT (CD117), CD34 and DOG-1. The malignancy potential of GIST was accessed on the basis of tumor size and mitotic index under the 50 high power fields (HPF). Leiomyoma was diagnosed by the positive reaction of smooth muscle actin and desmin.

Statistical analysis. The follow-up endoscopy was performed at the 1st and 3rd month after the procedure, in order to record any ESD-related complications, tumor residue, recurrence or metastasis. Statistical analyses were conducted using SPSS 21.0 statistics software (IBM Corp.). All data were analyzed as the means with SD (standard deviation).

\section{Results}

Baseline characteristics of eligible patients. Eight consecutive patients (median age: 53.5 years; range: 22.0-78.0 years) fulfilled the entry criteria and underwent the advanced method of ESD. There were 3 males and 5 females. Three lesions were located in subcardia region and 5 were in the fundus. There was no sign of metastasis in these patients. The EUS findings showed that all tumors had a connection with the fourth EUS layer, indicating that these tumors originated from the muscularis propria.

Outcomes of endoscopical resection. ESD using the advanced procedure was carried out successfully in all eight patients with a mean operation time of $83 \pm 13 \mathrm{~min}$ (range: $60-100 \mathrm{~min}$ ). The 
Table II. Histopathological characteristics of gastric subepithelial tumors.

\begin{tabular}{ll}
\hline Patients & No. \\
\hline Size (pathology) & \\
$<4.5 \mathrm{~cm}$ & 2 \\
$4.5-5.5 \mathrm{~cm}$ & 5 \\
$>5.5 \mathrm{~cm}$ & 1 \\
Pathological diagnosis & \\
Leiomyomas & 6 \\
GI stromal tumors & 2 \\
Risk classification & \\
Low risk & 1 \\
Moderate risk & 0 \\
High risk & 1 \\
Immunohistochemistry data & \\
CD117 $^{+}$ & 2 \\
CD34 $^{+}$ & 2 \\
DOG-1 & \\
SMA $^{+}$ & 2 \\
Desmin $^{+}$ & 6 \\
\hline
\end{tabular}

mean tumor size was $45.6 \pm 7.5 \mathrm{~mm}$ (range: $40.0-65.0 \mathrm{~mm}$ ). The en bloc resection rate was $100 \%$ in these patients, with $87.5 \%$ complete resection rates. The average numbers of forceps were 12 (range: 7-20). Although perforations occurred in 5 out of 8 patients, all of them were positive perforations by EFR technique, and all were successfully closed with endoclips. No patient presented tension pneumoperitoneum, and all patients with perforations received gastrointestinal decompression after the procedure. The estimated blood loss was $20-100 \mathrm{ml}$, and no patient required blood transfusion. None of the patients needed further gastrectomy and no other complications were recorded. Table I summarizes the baseline characteristics and the outcomes of the endoscopic resection.

Histopathological findings. The average size of lesions were $48.5 \pm 8.0 \mathrm{~mm}$ (range: $40.0-66.0 \mathrm{~mm}$ ) in diameter. Six of the patients were diagnosed as leiomyomas, and two were GI stromal tumors. No patients had lymph node metastasis. The immunohistochemistry staining revealed that the positive rates of c-KIT (CD117), CD34 and DOG-1, SMA, Desmin were $25,25,25,75,75 \%$, respectively. One patient was at low risk of malignant potential, none was at intermediate risk and one patient was at high risk. Table II summarizes the histopathological evaluation of the resected specimens.

The median follow-up was 36 months (range: $28-54$ months), and no patient developed local recurrence or distant metastasis. The average length of inpatient hospital stay was 10 days (range: 6-16 days).

\section{Discussion}

Most subepithelial tumors are asymptomatic and are usually diagnosed incidentally during endoscopy. There are various types of tumors, including GIST, lipomas, leiomyomas, schwannomas and non-GIST sarcomas $(2,11)$. GISTs are the most common gastric mesenchymal neoplasms, but some might have been previously misdiagnosed as leiomyomas during endoscopical examination (12). Among the 8 patients in our study, 2 cases were misclassified as leiomyomas but were finally confirmed as GISTs according to histopathological results. This emphasizes the importance of immunohistochemistry staining in the final diagnosis.

Over the past decades, open surgery has been the major treatment option of removing subepithelial tumors $(3,13,14)$, especially for those with large tumors in the subcardia region. Although surgery can provide satisfactory clinical outcomes, the proximal resection of the stomach might results in severe gastroesophageal reflux disease and stenosis, which, in the long run, reduces the quality of life among patients $(15,16)$.

Laparoscopic surgery and endoscopic resection have emerged in recent years as alternative treatments. According to the latest guidelines in Asian countries $(17,18)$, laparoscopic technique is only recommended for small GISTs (no more than $5 \mathrm{~cm}$ ) and is associated with the possibility of abdominal metastasis (19). As a newly developed treatment, endoscopic resection (ER) has become a minimally invasive treatment for gastric subepithelial tumors (5-7,9-11). The implementation of this technique facilitates the preservation of organ and functions in the GI tract, however, the safety and endoscopical outcomes still remain uncertain.

Due to the possibility of procedure-related complications and some technical limitations especially for tumors in the subcardia region $(5,6,20)$ and to the anatomic feature of the cardia where the His angle is very sharp and the blood supply is abundant, it might be technically difficult to perform endoscopical treatment even for experienced endoscopists. Traditional ESD procedure includes the circumferential cutting of the mucosa surrounding the tumor followed by submucosal dissection $(5,9,10)$. If a tumor is large, all the overlying mucosa will be removed after resection, which makes the closure of residual tissue much more difficult $(6,21)$. Moreover, formation of the scar will result in preventricular stenosis, which influences the function of the gastric cardia.

To improve the feasibility of ESD in removing large SMTs around gastric cardia, we made the following technical adjustments: firstly, different from the conventional ESD procedure, our method omitted the step of marking the outside borders of the tumor, and a saline solution was directly injected around the lesion to lift it off the muscular layer. This simplified the whole procedure and relatively reduced the operation time; secondly, instead of circumferential incision around the lesion, we initially cut along the mucosa with a median linear incision in central region of the tumor. The purpose of this step was to reserve the overlying mucosa and thus guarantee the successful closure of residual defect after tumor dissection. Furthermore, after the median linear incision of the mucosa was made in the central area of the tumor, the submucosal tissues were dissected gradually from both sides of the incision, this facilitated the exposure of the tumor and thus made the dissection easier than before.

The major advantage of our procedure was that it simplified the traditional ESD method and facilitated the application of ESD in large SMTs. In general, perforation was considered 
a severe ESD-related complication, which mainly restricted the application of ESD. However, with the development of endoscopic full-thickness resection (EFR) $(11,22,23)$, the positive perforation facilitated the removal of tumors, especially for those with a deep invasion of the muscularis propria layer or even the serosa. In our study, although the perforation rate was $75 \%$, all of them were positive perforations and tumors were successfully removed using the EFR.

The closure technique remains one of the most important steps after dissection of tumors. Endoclips are generally used in traditional EFR for small defects. If the tumor is large in size, air suction will be applied to reduce the defect $(24,25)$. Although new techniques such as the omental patch method and string suture have been used to close large defects, they are time-consuming and require greater technical skills than endoclips-assisted closure. Differently, in our method, the closure step is easily conducted by using several endoclips because we initially preserved the overlying mucosa. Neither omental patch method nor string suture is needed in our procedure, and there is no necessity to perform the 'suction-clip-suture', which dramatically simplifies the closure procedure. During the short-term follow-up, no remarkable complications were recorded in any of the patients.

In conclusion, the advanced method of ESD is a feasible and safe procedure for tumors especially for large tumors located in subcardia region. It could be applied as a novel technique for treating patient without performing open gastrectomy or any laparoscopic-assisted techniques.

\section{Acknowledgements}

Not applicable.

\section{Funding}

No funding was received.

\section{Availability of data and materials}

The datasets used and/or analyzed during the current study are available from the corresponding author on reasonable request.

\section{Authors' contributions}

$\mathrm{BL}$ wrote the manuscript. $\mathrm{HC}$ and $\mathrm{WZ}$ collected and analyzed the general data of patients. BL and GZ were responsible for the analysis of the histopathology results. All the authors read and approved the final manuscript.

\section{Ethics approval and consent to participate}

The study was approved by the Ethics Committee of First Affiliated Hospital of Nanjing Medical University (Nanjing, China). Patients who participated in this research had complete clinical data. Signed informed consents were obtained from the patients or the guardians.

\section{Patient consent for publication}

Not applicable.

\section{Competing interests}

The authors declare that they have no competing interests.

\section{References}

1. Salah W and Faigel DO: When to puncture, when not to puncture: Submucosal tumors. Endosc Ultrasound 3: 98-108, 2014.

2. Janeway KA and Weldon CB: Pediatric gastrointestinal stromal tumor. Semin Pediatr Surg 21: 31-43, 2012.

3. Nishida T, Blay JY, Hirota S, Kitagawa Y and Kang YK: The standard diagnosis, treatment, and follow-up of gastrointestinal stromal tumors based on guidelines. Gastric Cancer 19: 3-14, 2016.

4. Hirao M, Masuda K, Asanuma T, Naka H, Noda K, Matsuura K, Yamaguchi $\mathrm{O}$ and Ueda N: Endoscopic resection of early gastric cancer and other tumors with local injection of hypertonic saline-epinephrine. Gastrointest Endosc 34: 264-269, 1988.

5. Lee IL, Lin PY, Tung SY, Shen CH, Wei KL and Wu CS: Endoscopic submucosal dissection for the treatment of intraluminal gastric subepithelial tumors originating from the muscularis propria layer. Endoscopy 38: 1024-1028, 2006.

6. Chung IK, Lee JH, Lee SH, Kim SJ, Cho JY, Cho WY, Hwangbo Y, Keum BR, Park JJ, Chun HJ, et al: Therapeutic outcomes in 1000 cases of endoscopic submucosal dissection for early gastric neoplasms: Korean ESD Study Group multicenter study. Gastrointest Endosc 69: 1228-1235, 2009.

7. Jang YS, Lee BE, Kim GH, Park DY, Jeon HK, Baek DH, Kim DU and Song GA: Factors associated with outcomes in endoscopic submucosal dissection of gastric cardia tumors: A retrospective observational study. Medicine (Baltimore) 94: e1201, 2015.

8. McColl KE: Cancer of the gastric cardia. Best Pract Res Clin Gastroenterol 20: 687-696, 2006

9. Kakushima N, Yahagi N, Fujishiro M, Kodashima S, Nakamura M and Omata M: Efficacy and safety of endoscopic submucosal dissection for tumors of the esophagogastric junction. Endoscopy 38: 170-174, 2006

10. Białek A, Wiechowska-Kozłowska A, Pertkiewicz J, Polkowski M, Milkiewicz P, Karpińska K, Ławniczak M and Starzyńska T: Endoscopic submucosal dissection for treatment of gastric subepithelial tumors (with video). Gastrointest Endosc 75: 276-286, 2012.

11. Zhou PH, Yao LQ, Qin XY, Cai MY, Xu MD, Zhong YS, Chen WF, Zhang YQ, Qin WZ, Hu JW, et al: Endoscopic fullthickness resection without laparoscopic assistance for gastric submucosal tumors originated from the muscularis propria. Surg Endosc 25: 2926-2931, 2011.

12. Kang HC, Menias CO, Gaballah AH, Shroff S, Taggart MW, Garg N and Elsayes KM: Beyond the GIST: Mesenchymal tumors of the stomach. Radiographics 33: 1673-1690, 2013.

13. Nishida T, Hirota S, Yanagisawa A, Sugino Y, Minami M, Yamamura Y, Otani Y, Shimada Y, Takahashi F and Kubota T; GIST Guideline Subcommittee: Clinical practice guidelines for gastrointestinal stromal tumor (GIST) in Japan: English version. Int J Clin Oncol 13: 416-430, 2008.

14. Roggin KK and Posner MC: Modern treatment of gastric gastrointestinal stromal tumors. World J Gastroenterol 18: 6720-6728, 2012.

15. Kong SH and Yang HK: Surgical treatment of gastric gastrointestinal stromal tumor. J Gastric Cancer 13: 3-18, 2013.

16. Kim JW, Yoon H, Kong SH, Kim JS, Paeng JC, Lee HJ, Lee KU and Yang HK: Analysis of esophageal reflux after proximal gastrectomy measured by wireless ambulatory $24-\mathrm{hr}$ esophageal $\mathrm{pH}$ monitoring and TC-99m diisopropyliminodiacetic acid (DISIDA) scan. J Surg Oncol 101: 626-633, 2010.

17. ESMO/European Sarcoma Network Working Group: Gastrointestinal stromal tumours: ESMO Clinical Practice Guidelines for diagnosis, treatment and follow-up. Ann Oncol 25 (Suppl 3): iii21-iii26, 2014.

18. Kang YK, Kang HJ, Kim KM, Sohn T, Choi D, Ryu MH, Kim WH and Yang HK; Korean GIST Study Group (KGSG): Clinical practice guideline for accurate diagnosis and effective treatment of gastrointestinal stromal tumor in Korea. Cancer Res Treat 44: 85-96, 2012.

19. Kim MD, Kang DH, Park JH, Lee JH, Choi CW, Kim DH, Kim HW and Kim GH: Abdominal wound metastasis after laparoscopic surgery of gastrointestinal stromal tumor. Gut Liver 4: 283-286, 2010. 
20. Kakushima N, Fujishiro M, Kodashima S, Muraki Y, Tateishi A, Yahagi $\mathrm{N}$ and Omata $\mathrm{M}$ : Technical feasibility of endoscopic submucosal dissection for gastric neoplasms in the elderly Japanese population. J Gastroenterol Hepatol 22: 311-314, 2007.

21. Imagawa A, Okada H, Kawahara Y, Takenaka R, Kato J, Kawamoto H, Fujiki S, Takata R, Yoshino T and Shiratori Y: Endoscopic submucosal dissection for early gastric cancer: Results and degrees of technical difficulty as well as success. Endoscopy 38: 987-990, 2006.

22. Shi Q, Chen T, Zhong YS, Zhou PH, Ren Z, Xu MD and Yao LQ: Complete closure of large gastric defects after endoscopic fullthickness resection, using endoloop and metallic clip interrupted suture. Endoscopy 45: 329-334, 2013.

23. Schlag C, Wilhelm D, von Delius S, Feussner H and Meining A: EndoResect study: Endoscopic full-thickness resection of gastric subepithelial tumors. Endoscopy 45: 4-11, 2013.
24. Chiu PW, Phee SJ, Wang Z, Sun Z, Poon CC, Yamamoto T, Penny I, Wong JY, Lau JY and Ho KY: Feasibility of fullthickness gastric resection using master and slave transluminal endoscopic robot and closure by overstitch: A preclinical study. Surg Endosc 28: 319-324, 2014.

25. Mori H, Kobara H, Rafiq K, Nishiyama N,Fujihara S, Kobayashi M, Oryu M, Fujiwara M, Suzuki Y and Masaki T: New flexible endoscopic full-thickness suturing device: A triple-arm-bar suturing system. Endoscopy 45: 649-654, 2013.

(6) (9) This work is licensed under a Creative Common Attribution-NonCommercial-NoDerivatives 4.0 International (CC BY-NC-ND 4.0) License. 